\title{
What's in the Game? Developing a Taxonomy of Gamification Concepts for Health Apps
}

\author{
Manuel Schmidt-Kraepelin \\ University of Kassel \\ $\underline{\text { schmidt-kraepelin@uni-kassel.de thiebes@uni-kassel.de }}$
}

\author{
Minh Chau Tran \\ University of Cologne \\ $\underline{\text { mtran1@smail.uni-koeln.de }}$ \\ Ali Sunyaev \\ University of Kassel \\ sunyaev@,uni-kassel.de
}

\begin{abstract}
Gamification is a promising approach to tackle users' infrequent and decreasing use of health apps. For this purpose, extant research provides developers of health apps with a vast number of different game elements. By abstracting from the implementation of single game elements and choosing a more holistic approach to gamification concepts, we iteratively develop a taxonomy of gamification concepts for health apps using inductive and deductive approaches and discuss its transferability to other gamification contexts. We contribute to a profound understanding of the main characteristics of gamification concepts and enable researchers and practitioners to classify and distinguish them. Our results provide interesting insights into the essential characteristics of health apps' gamification concepts.
\end{abstract}

\section{Introduction}

With the growing popularity of videogames and the rising app culture, the idea of 'gamifying' applications has turned out to be a promising approach to get users actively involved and keeping them engaged over a sustained period of time [16]. Since its introduction, the concept of gamification has been rapidly established and widely adopted by practitioners and researchers in various domains such as workplace, education, marketing, and healthcare [17]. The main purpose of deploying game elements in these non-game contexts is to reinforce desired behavioral traits [1]. In order to do so, gamification aims at making repetitive, tiresome everyday tasks more enjoyable by applying gamification elements that foster intrinsic motivation [44].

When researchers or practitioners decide to gamify an application, extant research provides them with a vast number of different game elements (e.g., points, leaderboards, avatars) that might help to achieve the desired behavioral outcomes [44]. However, meaningful gamification requires more than solely choosing and implementing some of the described game elements [18]. To make use of the full motivational potential of gamification, researchers and practitioners also need to consider the application context, characteristics of targeted users, effects arising from the interplay between different game elements, and their relationship to the desired application purpose. Thus, within this work we aim to abstract from the implementation of single game elements and call for a more holistic approach to the description and classification of gamification concepts.

We argue that the domain of health apps is particularly suitable to better understand the essential characteristics of gamification concepts for several reasons. First, health apps have great potential to significantly impact users' health-related behavior but often suffer from insufficient user motivation [25]. As a result, healthcare has become one of the main domains for research on and application of gamification concepts [17]. Second, health apps are highly diverse. Typical examples include apps that motivate users to be more physically active [8], apps that help to properly manage chronic diseases [42], and apps that aim to foster health professionals' compliance to hygiene standards [23]. For these reasons, we presume gamification concepts employed in healthcare to be more mature, professionalized, and diverse than in most other domains. To enable researchers and practitioners to describe and classify gamification concepts of health apps in a more holistic manner than solely describing implemented game elements, we seek to answer the following research question with this work: How can gamification concepts of health apps be classified?

To answer this research question, we propose a taxonomy of gamification concepts for health apps developed according to the taxonomy development method by Nickerson et al. [36]. By doing so, we aim to contribute to a profound understanding of gamification concepts and exhibit their essential characteristics. When developing our taxonomy, we abstract from the implementation of single game elements as we are interested in more general insights into characteristics of 
gamification concepts that help to classify and distinguish them. As gamification is a novel approach and research in this field is rather immature and low on theoretical insights [40], we think that the conceptual knowledge resulting from developing a taxonomy makes a meaningful contribution to the information systems (IS) community and can serve as a foundation for sense-making and theory-driven research on gamification in IS in the future.

Our study is structured as follows: First, we give a brief introduction to gamified health apps, taxonomies in IS research, and related classifications in the area of gamified health apps. We then introduce the research method we applied to develop our taxonomy. Third, we present our taxonomy of health app gamification concepts and outline its dimensions and characteristics. In addition, we outline the most interesting findings from the classification of 27 health app gamification concepts that were identified in literature and served as the basis for our inductive iterations during the taxonomy development. Finally, we discuss implications, limitations, and opportunities for future research before we end our paper with a brief conclusion.

\section{Related work}

\subsection{Gamified health apps}

With the potential to facilitate the management and prevention of diseases, supporting healthier lifestyles, and promoting health-related education, health apps have become a widely-recognized tool within the healthcare domain [28]. Thereby, health apps refer to all kinds of digital applications aiming to evoke any healthrelated outcome (e.g., physical activity, nutrition, rehabilitation, and education) in either a personal (wellness) or professional (healthcare) setting, including mobile and web-based health applications, as well as desktop health applications. An exemplary overview of gamified health apps can be found in [17]. In spite of health apps' potential to positively influence users' health-related behavior, users often stop using health apps due to a loss of interest and decreasing long-term motivation [25]. Techniques for the promotion of users' endurance to use health apps are crucial. Hence, gamification has gained importance in the healthcare domain as it is considered to be a promising approach to tackle users' infrequent and decreasing use of health apps [11].

Literature provides two prevailing definitions for gamification. On the one hand, Deterding et al. define gamification as "the use of game design elements in non-game contexts" [12]. On the other hand, Huotari and Hamari [19] refer to gamification as the process of enhancing services with motivational affordances for gameful experiences. Kari et al. [21] propose that these definitions arise from two different understandings of gamification (i.e., process view and experience view). While the process of gamification is concerning the "intentional use of different methods to gamify some certain aspect of use", the experience of gamification focuses on the gameful experience of the user that might arise from implemented game elements but can also emerge from non-gamified features [21]. We align our understanding of gamification with the view of Huotari and Hamari [19] and take an experience view of gamification [21]. We aim to use this view of gamification in order to abstract from the intentional use of single game elements to gamify certain aspects of use and call for a more holistic approach to gamification concepts within this work. We consider the gamification concept of a health app to be the entirety of design choices of gamified health apps that might invoke gameful experiences for its users and that address a certain health-related outcome, as well as the effects occurring from their interplay, and their relationship to the underlying desired health-related outcome.

\subsection{Taxonomies}

Classification systems are important tools in most scientific disciplines, including IS research [36]. They help researchers to understand and analyze complex domains. Within the IS discipline, taxonomies are the prevailing form of classification systems [36]. While the term taxonomy can describe both, the process of classifying objects as well as the resulting classification system itself [3], we align our understanding of what constitutes a taxonomy with Nickerson et al. [36]. who define a taxonomy $\mathrm{T}$ as a set of "[...] $\mathrm{n}$ dimensions $\mathrm{D}_{\mathrm{i}}$ $(i=1, \ldots, n)$ each consisting of $k_{i}\left(k_{i} \geq 2\right)$ mutually exclusive and collectively exhaustive characteristics $C_{i j}$ $\left(\mathrm{j}=1, \ldots, \mathrm{k}_{\mathrm{i}}\right)$ such that each object under consideration has one and only one $\mathrm{C}_{\mathrm{ij}}$ for each $\mathrm{D}_{\mathrm{i}}$."

Taxonomies structure and organize knowledge in a specific domain and allow for performing "ex post theory building" [4]. They are a form of conceptual knowledge that can aid in developing theories (i.e., descriptive knowledge) [20]. Thus, the development of taxonomies serves an important purpose in IS research, a field that is dominated by the creation of conceptual knowledge [20]. Extant literature has already proposed some taxonomies related to behavior change techniques (e.g., gamification) and healthcare. Sawyer, for instance, describes a taxonomy of "current and possible future activity areas for games for health" [41]. While this taxonomy consists of only two dimensions, it was further extended by McCallum [30] into a four- 
dimensional taxonomy on games for health. Both taxonomies' scope (i.e., gaming in healthcare in general [41] and serious games and gamification in healthcare [30]) is much broader than the focus of this research, with gamification being only one potential part of those taxonomies. Adding to this, literature provides a variety of different categorizations for game design elements on different levels of abstraction. For example, Deterding et al. categorize game design elements on five different levels (i.e., game interface design patterns, game design patterns and mechanics, game design principles and heuristics, game models, game design methods) [12]. Blohm and Leimeister, on the other hand, categorize different game design elements as game mechanics or game dynamics and match them with their underlying motives for implementation [5]. In addition, Robinson and Belloti provide a detailed categorization of gamification elements on three hierarchical levels with the following categories on the highest tier: General Framing, General Rules and Performance Framing, Social Features, Incentives, Resources and Constraints, and Feedback and Status Information [39]. While we acknowledge the importance and value of categorizations of game elements on different levels of abstraction, we aim to contribute to the scientific knowledge base by proposing a taxonomy that is not limited to single game elements, is explicitly build for the health context, and is consistent with the taxonomy definition by Nickerson et al. [36]. Thus, our taxonomy gives researchers and practitioners the opportunity to analyze and classify gamification concepts on a given set of dimensions and mutually exclusive characteristics and not only state whether certain game elements are implemented or not. Another interesting approach to classify gamification has been proposed by Raftopoulos and Walz [37]. In contrast to the approaches discussed before, their classification is not limited to game elements only, but also incorporates aspects like primary gamification purpose, target audience, and technological aspects. However, their classification is limited to the context of enterprise gamification and is not compliant with the definition of taxonomies proposed by Nickerson et al. [36] as their proposed characteristics are not mutually exclusive. Overall, there is to the best of our knowledge no classification of gamification concepts in health apps that consists of a set of dimensions with mutually exclusive and collectively exhaustive characteristics.

\section{Research approach}

\subsection{Phase 1 - Data collection}

In the first phase, we conducted a literature review oriented towards Webster and Watson [48]. The objective of this phase was to identify research articles that focus on gamified health apps, which we could use as a foundation for the taxonomy development in the second phase. We searched scientific literature databases EBSCOhost (Business Source Complete, Academic Search Complete, and MEDLINE), Science Direct, and PubMed for two mandatory groups of keywords in title, abstract, and keywords. First, every paper had to contain the word 'gamify*'. Second, in order to limit our search to health-related contexts, every paper had to contain at least one of the following words: 'health*', 'exer*' (in order to cover words like 'exercise' and 'exer-games'), 'medic*', 'therap*', 'life*', 'fitness'. Where possible, we limited our search to peer-reviewed articles and articles published in 2010 or later since the term gamification did not gain widespread recognition in research and practice until 2010 [28].

Table 1. Apps identified by the review.

\begin{tabular}{|l|l|l|}
\hline ID & Gamified Health App & Reference(s) \\
\hline APP-1 & My Diet Coach - Weight Loss & {$[29]$} \\
\hline APP-2 & Calorie Counter/My Fitness & {$[29]$} \\
& Pal & \\
\hline APP-3 & Diet Point - Weight Loss & {$[29]$} \\
\hline APP-4 & Lose Weight Without Dieting & {$[29]$} \\
\hline APP-5 & Health Heroes & {$[10]$} \\
\hline APP-6 & sjekkdeg.no & {$[14]$} \\
\hline APP-7 & bant & {$[7]$} \\
\hline APP-8 & The Heart Game & {$[13]$} \\
\hline APP-9 & Empower/Picture It! & {$[6]$} \\
\hline APP-10 & HealthSeeker & {$[6]$} \\
\hline APP-11 & Nike+ Fuel & {$[43]$} \\
\hline APP-12 & Fitocracy & {$[24]$} \\
\hline APP-13 & Lifestyle Tool & {$[45]$} \\
\hline APP-14 & ONESELF & {$[1,2]$} \\
\hline APP-15 & HealthyTogether & {$[1]$} \\
\hline APP-16 & Endomondo & {$[1]$} \\
\hline APP-17 & StepByStep & {$[1]$} \\
\hline APP-18 & Kaizen-IM & {$[35]$} \\
\hline APP-19 & (no name) & {$[26]$} \\
\hline APP-20 & (no name) & {$[23]$} \\
\hline APP-21 & The Challenger App & {$[32]$} \\
\hline APP-22 & SuperBetter & {$[31]$} \\
\hline APP-23 & Milk Man & {$[49]$} \\
\hline APP-24 & GOODcoins & {$[47]$} \\
\hline APP-25 & Snack Track School & {$[46]$} \\
\hline APP-26 & (no name) & {$[27]$} \\
\hline APP-27 & (no name) & {$[9]$} \\
\hline
\end{tabular}

Our search yielded a total of 229 unique research articles. Two researchers independently assessed all 
articles' eligibility by screening their title, abstract, and keywords. Differences in the researchers' assessments were discussed and final decision about the inclusion or exclusion of an article was made jointly. To be eligible for the second phase, articles had to provide a reasonable description of the gamification concept of at least one gamified health app $(n=21)$. We excluded articles that were not peer-reviewed $(n=66)$, not in English $(n=7)$, or completely off-topic $(n=135)$. Of the remaining 21 articles some described multiple apps, which lead to the identification of 27 gamified health apps summarized in Table 1. For each gamified health app, we noted down its name (if available), a brief description extracted from the research articles that discussed the respective app, as well as a link to the website or Apple App Store or Google Play Store in case this information was available.

\subsection{Phase 2 - Taxonomy Development}

The second phase constitutes the actual taxonomy development, where we applied the method of Nickerson et al. since the development of a taxonomy based on specific guidelines avoids ad hoc classifications and ensures the quality and applicability of the final taxonomy [48]. The iterative development process comprises seven steps, which we briefly describe in the following.

3.2.1. Meta-characteristic. First, a meta-characteristic needs to be defined (Step 1). During the taxonomy development process researchers might tend to consider a large number of unrelated characteristics in hope of the emergence of a random pattern [36]. The definition of a meta-characteristics serves to avoid such situations by predefining an overall theme or rule that helps to eliminate irrelevant characteristics and identify new, but relevant characteristics. Thereby, the "metacharacteristic is the most comprehensive characteristic that will serve as the basis for the choice of characteristics in the taxonomy" [36]. Accordingly, each dimension and each characteristic is a "logical consequence of the meta-characteristic" [36]. For this research, we define our meta-characteristic to be characteristics of gamification concepts for health apps.

3.2.2. Ending conditions. The iterative nature of the taxonomy development process requires predefined conditions to terminate the process (Step 2). Nickerson et al. [36] provide five subjective and eight objective ending conditions, which we also adopt for this research. The subjective ending conditions are: (1) Conciseness: To ensure ease of use and thus avoid overwhelming researchers, the taxonomy should only contain a limited number of dimensions and characteristics in each dimension. (2) Robustness: The taxonomy should contain sufficient dimensions and characteristics, enabling to delineate objects and to differentiate them from each other. (3) Comprehensiveness: An empirically developed taxonomy should include classifications of all objects of the domain under consideration, whereas a conceptually developed taxonomy should contain all dimensions of the objects of interest. (4) Extendibility: The taxonomy must be adaptable to continuous changes and development in the domain by supporting the inclusion of additional dimensions and characteristics of new types of the objects. (5) Explanatory: The taxonomy should be explanatory as opposed to descriptive. It is not the objective of a taxonomy to delineate and encompass every single detail of the objects. The taxonomy rather serves to aid in understanding the objects in complex areas.

Since these subjective ending conditions are influenced by personal perceptions, additional objective ending conditions are required, which allow for unbiased decision whether to stop the iterative development process. They are: (1) All objects of interest or a representative sample thereof have been examined. (2) No object was merged with a similar object or split into multiple objects in the last iteration. A merge or split might affect changes to the objects. Thus, review of the corresponding dimensions and characteristics is necessary. (3) At least one object is classified under every characteristic of every dimension. If a characteristic without a matching object exists, there is a 'null' characteristic in the taxonomy. Therefore, it is necessary to identify at least one object for the characteristic, otherwise the characteristic needs to be removed. (4) No new dimensions or characteristics were added in the last iteration. If new dimensions or characteristics were found, it may be possible to find additional dimensions or characteristics in the following iteration. (5) No dimensions or characteristics were merged or split in the last iteration. A merge or split might affect changes to dimensions and characteristics. Thus, review of the other dimensions and characteristics is necessary. If required, a merge or split thereof needs to be performed. (6) Every dimension is unique and not repeated. If redundancies among dimensions exist in the taxonomy, they need to be removed to maintain the uniqueness of the taxonomy. (7) Every characteristic is unique within its dimension. If redundancies among characteristics exist in the taxonomy, they need to be removed to maintain the uniqueness of the taxonomy. (8) Each cell (i.e., combination of characteristics) is unique and is not repeated. If redundant cells exist in the taxonomy, they need to be removed to maintain the uniqueness of the taxonomy. 
3.2.3. Iteration. While most taxonomies in IS are developed either in an unsystematic manner or only inductively, respectively only deductively [36], the method of Nickerson et al. [36] includes an empiricalto-conceptual (inductive) as well as a conceptual-toempirical (deductive) approach. Consequently, prior to each iteration an approach has to be chosen by the researchers (Step 3). For the inductive approach, a subset of objects, which can be a random or systematic sample of objects, needs to be selected (Step 4e). Here, our overall sample of objects comprises a total of 27 gamified health apps, which we identified during the first phase. For each inductive approach, we randomly selected two to five apps out of this overall app sample. We chose to include two to five apps in each iteration to reduce the workload for a single iteration to a manageable amount as well as to include all apps described by a single study. Next, two researchers jointly examined, compared, and grouped selected objects in order to derive common characteristics (Step 5e). Finally, a preliminary taxonomy was created by grouping identified characteristics into dimensions (Step 6e). For the deductive approach, dimensions and characteristics of objects were derived based on the researchers' experience and extant knowledge on gamified health apps as well as from concepts in the studies that were reviewed during the first phase (Step 4c). Thereafter, newly conceptualized dimensions and characteristics were examined for their validity by reclassifying already classified objects based on these new dimensions and characteristics (Step 5c). Lastly, a (preliminary) taxonomy was formed by grouping the newly conceptualized dimensions and or characteristics into an initial taxonomy or adding them to an already existing taxonomy (Step 6c). For both, the inductive and the deductive approach, it had to be checked whether the (preliminary) taxonomy met the objective and subjective ending conditions after Step 6e/c (Step 7). If the ending conditions were not yet met, steps 3 to 6 were repeated. As soon as the objective and subjective ending conditions were met, the taxonomy development process terminated. Appendix A provides an overview of the iterations that were performed during this study.

\section{Taxonomy of gamification concepts for health apps}

\subsection{Overview}

In the following, we describe our taxonomy of gamification concepts for health apps'. In total, our taxonomy consists of 12 dimensions, each having two to three mutually exclusive characteristics. It must be noted that the purpose of our taxonomy was not to provide a classification of gamified health apps in general but rather a classification of gamification concepts implemented in such health apps. Thus, we abstracted from the technical perspective and did, for example, not include any dimension related to the devices an app runs on. We also abstracted from single game elements and instead focused more on the interaction between the gamification concept and app users. Figure 1 provides an overview of the final taxonomy. A detailed description of each dimension and its characteristics is given in the following section.

\subsection{Dimensions}

4.2.1. Gamification concept-to-user communication. How does the gamification concept communicate with the user? Gamification concepts do either directly convey messages to their users via textual or audio outputs or make use of some sort of mediator (e.g., an avatar interacting with the user).

4.2.2. User identity. How is the users' identity represented in the gamification concept? In some gamification concepts, users can create a unique virtual character (e.g., visually represented as an avatar) that can be customized over time as the user progresses within the gamification concept. Thereby, avatars related to user identities have to be distinguished from avatars that are independent from user identities, as is the case in the previously described gamification concept-to-user communication dimension. In other gamification concepts, users only choose a static selfselected identity (e.g., a nickname and a picture).

4.2.3. Rewards. Which rewards can users earn by playing and progressing within the gamification concept? Whereas some gamification concepts solely offer internal (virtual) rewards (e.g., badges) that are accessible only within the gamification concept itself, others additionally offer external (real-world) rewards such as discounts on real-world purchases. Some gamification concepts do not offer any rewards.

4.2.4. Competition. How do users compete with each other within the gamification concept? Some gamification concepts give users the opportunity to directly compete with each other on specific tasks. In this form of competition both users take on the same challenge or task and directly compare their performance. In other gamification concepts, users can compare their overall performance within the gamification concept with all other users (e.g., via point systems and leaderboards). We refer to this type of competition as indirect. Some gamification concepts do not offer any form of competition between users. 


\begin{tabular}{|c|c|c|c|}
\hline Dimension & \multicolumn{3}{|c|}{ Characteristics } \\
\hline $\begin{array}{l}\text { Gamification concept-to-user } \\
\text { communication }\end{array}$ & \multirow{2}{*}{\multicolumn{2}{|c|}{ Direct }} & Mediated \\
\hline User identity & & & Self-selected \\
\hline Rewards & Internal & Internal and external & No \\
\hline Competition & Direct & Indirect & No \\
\hline Target group & Patients & Healthy individuals & Health professionals \\
\hline Collaboration & Cooperative & Supportive only & No \\
\hline Goal-setting & \multicolumn{2}{|l|}{ Self-set } & Externally set \\
\hline Narrative & \multicolumn{2}{|l|}{ Continuous } & Episodical \\
\hline Reinforcement & \multicolumn{2}{|l|}{ Positive } & Positive-negative \\
\hline Level of integration & \multicolumn{2}{|l|}{ Independent } & Inherent \\
\hline Persuasive intent & Compliance change & Behavior change & Attitude change \\
\hline User advancement & Presentation only & Progressive & No \\
\hline
\end{tabular}

Figure 1. Final taxonomy of gamification concepts for health apps.

4.2.5. Target group. Who is targeted to use the gamification concept? For health apps, the target group of the gamification concept can either be patients (i.e., the app aims to help its users in healing from a specific disease), healthy individuals (i.e., users are not suffering from a specific disease targeted by the app, but want to improve their overall health status and well-being), or health professionals. It is important to notice that one should differentiate between the target group of the health app itself and the target group of the gamification concept. Although these might be identical for many health apps, in other cases the target group of the gamification concept might only be a subgroup of the health app target group (e.g., an app in which a health professional provides content for patients but the gamification concept only targets patients).

4.2.6. Collaboration. Which form of collaboration does the gamification concept offer? Some gamification concepts only offer what we refer to as supportive collaboration. In this form of collaboration, users can be motivated by other people who are not necessarily users of the app (e.g., via social networks). Other gamifications concepts give users the opportunity to actively cooperate on a specific task or challenge within the gamification concept, thus cooperatively contributing to its accomplishment. Some gamification concepts do not offer any form of collaboration.

4.2.7. Goal setting. Who sets goals within the gamification concept? In some gamification concepts, users set the goals they want to reach on their own (either completely free or by selecting goals from a predefined list of goals provided by the app). In other gamification concepts, goals are prescribed by an external source (e.g., by the app developer or a health professional).

4.2.8. Narrative. How does the gamification concept behave over time? The gamification concept can be either episodical (i.e., the gamification concept is clearly divided into different stages and/or the user progress is partially or fully reset after a certain amount of time) or continuous (i.e., the gamification concept is not divided into distinguishable stages and user progress is never reset).

4.2.9. Reinforcement. How does the gamification concept attempt to reinforce its users? Gamification concepts do either only use positive reinforcement (e.g., by highlighting current and future successes) or additionally use negative reinforcement (e.g., by referring to failures from the past or portending to deduct points).

4.2.10. Persuasive intent. Which type of health-related change does the gamification concept aim to evoke? Health app gamification concepts do either aim to evoke a compliance change (i.e., being compliant to very specific rules or guidelines), a behavioral change (i.e., fostering health-promoting behavior in a specific context without strict rules or guidelines), or attitude change (i.e., influencing the users' fundamental attitude towards a certain health-related topic) [1].

4.2.11. Level of integration. To which extent is the gamification concept cohesively related to the underlying health-related activities? Gamification concepts can be either independent (i.e., the gamification concept is superficially implemented and 
the underlying health-related activities could be easily performed without the gamification concept) or inherent (i.e., the health-related activity is partially or fully embedded in the gamification concept), for example, by dividing the activity into missions [34].

4.2.12. User advancement. How does the gamification concept consider the overall user advancement? The overall user advancement can either only be presented to the user (e.g., via experience points or progress bars) or the gamification concept can in addition utilize users' progress to adapt the gamification content to users' skills (e.g., unlocking higher difficulty levels when a user has gathered a certain amount of experience points). Some gamification concepts do not consider the users' overall advancement in any way.

\section{Discussion}

Analysis of our taxonomy and the classification of examined objects reveals some interesting insights into the current landscape of gamification concepts for health apps. Out of 27 apps, 21 used positive reinforcement only, whereas 6 apps used a combination of positive and negative reinforcement. We did not find any health app that solely relied on negative reinforcement. Given the serious context, that is healthcare, and the risks of potentially evoking detrimental effects through negative reinforcement [22], this comes as no surprise. One examined app, for example, aimed to support patients in overcoming their social anxiety [32]. Especially for such patients, negative reinforcement might have detrimental effects. Overall, the application of negative reinforcement techniques might be more suitable for less serious contexts. Another interesting finding concerns the fact that although potentially possible, no app used a combination of indirect and direct competition. In general, apps seemed to favor indirect competition over direct competition, which could have diverse reasons such as implementation efforts. Again, the health context might be another reason for the low number of apps implementing direct competition since competition could be viewed inappropriate for many healthcare settings, especially in cases where patients are involved.

From our point of view, external rewards can, in some cases, make a useful supplement to gamification concepts for health apps. However, gamification should always focus on fostering intrinsic motivation and developers need to be careful that external rewards do not prevail as intrinsic motivation bears higher potential for sustained behavioral influence [15]. Thus, it is no surprise that no app solely relied on external rewards.

Looking at our object classifications, we find that only 6 apps made use of episodical narratives. However, we think that episodical narratives can be beneficial for many gamified health apps as they counteract the risk of declining positive effects over time, which might occur when the novelty of gamification has worn off [33]. Online games like Diablo 3 by Activision Blizzard successfully use this approach and regularly reset the progress of their players. By doing so, they motivate players to return to the game for a certain amount of time and lower the entry threshold for new players.

Within our taxonomy we distinguish compliance, behavioral, and attitude changes in the persuasive intent dimension. Most classified objects intended to evoke either compliance or behavioral changes, whereas only five objects intended to evoke an attitudinal change. Overall, this is not surprising since gamification is particularly suitable to promote compliance and behavioral change and its effect on attitudinal change has not yet been profoundly researched. Thus, researcher should further investigate the potential of gamified health apps for supporting attitudinal changes.

Our research contributes to scientific knowledge base in several aspects. By developing a taxonomy in line with the method proposed by Nickerson et al. [36], we shed some light on the characteristics of gamification concepts implemented in health apps and thereby contribute to the conceptual knowledge of gamification in healthcare. In addition, we promote a more holistic approach to gamification and ensure explanatory power of our taxonomy by abstracting from the implementation of single game elements and describing characteristics of gamification concepts as a whole. With this regard, future research should investigate potential interdependencies between some dimensions of our taxonomy. While in principle such interdependencies should be avoided during the development of a taxonomy [36], they might nonetheless be present in the objects to be classified. This could hint to the existence of archetypes of objects (i.e., archetypes of gamification concepts), thus further extending the explanatory power of our taxonomy. Finally, although our taxonomy is informed by a health app viewpoint, we think that it is partially transferable to other contexts as target group and persuasive intent are the only dimensions that are inseparably connected to the healthcare context. However, as our taxonomy has been exclusively build by analyzing gamification concepts from the health context, the inclusion of other gamification concepts might lead to additional dimensions and characteristics. From our point of view, it would be exciting to see whether our taxonomy could be utilized in other gamification contexts and whether objects from other contexts would exhibit different characteristics.

Although we used the user-centered experience view on gamification to develop our taxonomy, the results of 
our study can also be valuable for practical purposes as they provide practitioners with characteristics of gamification concepts in health apps and give developers the opportunity to reflect and further develop their own gamification concepts. We will aim to further extend this practical value in future research by using our taxonomy to identify archetypes of gamification concepts (in accordance with the approach by [38]) and investigate their relationship to underlying desired health-related outcomes. By doing so, we aim to provide practitioners with state-of-the-art gamification concepts for certain desired health-related outcomes.

Limitations of this study are as follows. First, our sample of objects is limited to apps that were discussed in literature. It is without question that there exist many more gamified health apps that are not covered by scientific literature. These apps might implement different gamification concepts, which could in turn lead to changes of our taxonomy. Future research should therefore investigate our taxonomy's applicability to such apps. Second, although our extensive literature review served to establish a comprehensive pool of gamified health apps for the taxonomy development, we cannot rule out the possibility that we missed some articles providing descriptions of gamified health apps. Nonetheless, we are confident that our list of apps constitutes a representative sample of apps discussed in the literature. Lastly, some app descriptions provided only limited or fuzzy information for some aspects covered by our taxonomy. It is thus possible that some of our object classifications are not entirely appropriate. We aimed to overcome such information deficits by triangulating our data and referring to additional information available in the app stores or app websites.

\section{Conclusion}

Gamification of health apps is a promising approach to counteract the often decreasing long-term motivation of health app users. Despite rapid adoption of gamification by practitioners and researchers in the healthcare domain, to date there is little knowledge on the actual gamification concepts implemented in such apps, that goes beyond single game elements. With this research, we have tried to shed light on this intriguing, yet largely unexplored area. Our main contribution is a taxonomy of gamification concepts for health apps that consists of twelve dimensions, each having between two and three characteristics. Furthermore, our findings highlight some interesting avenues for research and practice to explore novel gamification concepts for health apps.

\section{References}

[1] Alahäivälä, T. and Oinas-Kukkonen, $H$. Understanding Persuasion Contexts in Health Gamification: A Systematic Analysis of Gamified Health Behavior Change Support Systems Literature. International Journal of Medical Informatics, 96, 2016, pp. 62-70.

[2] Allam, A., Kostova, Z., Nakamoto, K. and Schulz P.J. The Effect of Social Support Features and Gamification on a Web-Based Intervention for Rheumatoid Arthritis Patients: Randomized Controlled Trial, Journal of Medical Internet Research, 17, 2015, pp. e14.

[3] Bailey, K.D., Typologies and Taxonomies: An Introduction to Classification Techniques, Sage, Thousand Oaks, CA, 1994.

[4] Bapna, R., Goes, P., Gupta, A. and Jin, Y. User Heterogeneity and Its Impact on Electronic Auction Market Design: An Empirical Exploration, MIS Quarterly, 28, 2004, pp. 21-43.

[5] Blohm, I. and Leimeister, J.M., Design of IT-Based Enhancing Services for Motivational Support and Behavioral Change, Business \& Information Systems Engineering, 2013, pp. 275-278.

[6] Boulos, M.N.K., Gammon, S., Dixon, M.C., MaxRury, S.M., Fergusoon M.J., Rodrigues, F.M., Baptista, T.M. and Yang, S.P. Digital Games for Type 1 and Type 2 Diabetes: Underpinning Theory with Three Illustrative Examples, JMIR Serious Games, 3, 2015, pp. e3.

[7] Cafazzo, J.A., et al., "Design of an Mhealth App for the Self-Management of Adolescent Type 1 Diabetes: A Pilot Study", Journal of Medical Internet Research, 14, 2012, pp. e70.

[8] Chung, A.E., Casselmann, M., Hamming, N., Katzman, D.K. and Palmert, M.R. Tweeting to Health: A Novel Mhealth Intervention Using Fitbits and Twitter to Foster Healthy Lifestyles", Clinical Pediatrics, 56, 2017, pp. 26-32.

[9] Cudney, E.A., Murray, S.L., Sprague, C.M., Byrd, L.M., Morris, F.M., Merwin, N. and Warner, D.L. Engaging Healthcare Users through Gamification in Knowledge Sharing of Continuous Improvement in Healthcare, Procedia Manufacturing, 3, 2015, pp. 34163423.

[10] Curtis, K.E., Lahiri, S. and Brown, K.E. Targeting Parents for Childhood Weight Management: Development of a Theory-Driven and User-Centered Healthy Eating App", JMIR mHealth and uHealth, 3(2), 2015, pp. e69.

[11] Dennison, L., Morrison, L., Conway, G. and Yardley, L., Opportunities and Challenges for Smartphone Applications in Supporting Health 
Behavior Change: Qualitative Study, Journal of Medical Internet Research, 15(4), 2013, pp. e86.

[12] Deterding, S., Dixon, D., Khaled, R. and Nacke, L. From Game Design Elements to Gamefulness: Defining Gamification, Proceedings of the 15th international academic MindTrek conference: Envisioning future media environments, Tampere, Finland, Sep 28-30 2011, pp. 9-15.

[13] Dithmer, M. Rasmusen, J.O., Grönvall, E., Spindler, H., Hansen, J., Nielsen, G., Sorensen, S.B. and Dinesen, B., "The Heart Game": Using Gamification as Part of a Telerehabilitation Program for Heart Patients, Games for Health Journal, 5(1), 2016, pp. 27-33.

[14] Gabarron, E., Schopf, T.R., Serrano, J.A., Fernandez-Luque, L. and Dorronzoro, E. Gamification Strategy on Prevention of STDs for Youth, Studies in Health Technology and Informatics, 192, 2013.

[15] Gnauk, B., Dannecker, L. and Hahmann, M. Leveraging Gamification in Demand Dispatch Systems, in Proceedings of the 2012 Joint EDBT/ICDT Workshops, Berlin, Germany, 2012, pp. 103-110.

[16] Hamari, J., Transforming Homo Economicus into Homo Ludens: A Field Experiment on Gamification in a Utilitarian Peer-to-Peer Trading Service, Electronic Commerce Research and Applications, 12(4), 2013, pp. 236-245.

[17] Hamari, J. and Koivisto, J., "Working out for Likes": An Empirical Study on Social Influence in Exercise Gamification, Computers in Human Behavior, 50, 2015, pp. 333-347.

[18] Hamari, J., Koivisto, J. and Sarsa, H. Does Gamification Work?--a Literature Review of Empirical Studies on Gamification, 47th Hawaii International Conference on System Sciences, Waikoloa, HI, Jan 6-9 2014, pp. 3025-3034.

[19] Huotari, K. and Hamari, J. Defining Gamification: A Service Marketing Perspective, Proceedings of the 16th International Academic MindTrek Conference, Tampere, Finland, Oct 3-5. 2012, pp. 17-22.

[20] Iivari, J., A Paradigmatic Analysis of Information Systems as a Design Science, Scandinavian Journal of Information Systems, 19(2), 2007, pp. 39-64.

[21] Kari, T., Piipo, J., Frank, L., Makkonen, M., Moilanen, P. To Gamify or Not to Gamify?: Gamification in Exercise Applications and Its Role in Impacting Exercise Motivation, Proceedings of the 29th Bled eConference, 2016, pp. 393-405.

[22] Kazdin, A.E., Acceptability of Aversive Procedures and Medication as Treatment Alternatives for Deviant Child Behavior, Journal of Abnormal Child Psychology, 12(2), 1984, pp. 289-301.

[23] Klein, F., Severijns, C., Albiez, D., Jovanovic, M. and Eyvazy Hesar, M. The Hygiene Games, Proceedings of the 13th International Congress in Nursing Informatics, 2016.
[24] Koivisto, J. and Hamari, J., Demographic Differences in Perceived Benefits from Gamification, Computers in Human Behavior, 35, 2014, pp. 179-188. [25] Krebs, P. and Duncan, D.T., Health App Use among US Mobile Phone Owners: A National Survey, JMIR mHealth and uHealth, 3(4), 2015, pp. e101.

[26] Lapão, L.V., Marques, R., Gregorio, J., Pinheiro, F., Povoa, P. and Mira Da Silva, M. Using Gamification Combined with Indoor Location to Improve Nurses' Hand Hygiene Compliance in an Icu Ward, Transforming Healthcare with the Internet of Things: Proceedings of the EFMI Special Topic Conference Paris, France Apr 17-19, 2016, pp. 3.

[27] Lin, R.J. and Zhu, X., Leveraging Social Media for Preventive Care-a Gamification System and Insights, Studies in Health Technology and Informatics, 180, 2011, pp. 838-842.

[28] Lister, C., West, J.H., Cannon, B., Sax, T. and Brodegard, D. Just a Fad? Gamification in Health and Fitness Apps, JMIR Serious Games, 2(2), 2014, pp. e9. [29] Maturo, A. and Setiffi, F. The Gamification of Risk: How Health Apps Foster Self-Confidence and Why This Is Not Enough, Health, Risk \& Society, 17(78), 2016, pp. 477-494.

[30] McCallum, S., Gamification and Serious Games for Personalized Health, Studies in Health Technology and Informatics, 177, 2012, pp. 85-96.

[31] Miller, A.S., Cafazzo, J.A. and Seto, E. A Game Plan: Gamification Design Principles in Mhealth Applications for Chronic Disease Management, Health Informatics Journal, 22(2), 2016, pp. 184-193.

[32] Miloff, A., Marklund, A. and Carlbring, P. The Challenger App for Social Anxiety Disorder: New Advances in Mobile Psychological Treatment, Internet Interventions, 2(4), 2015, pp. 382-391.

[33] Nakajima, T. and Lehdonvirta, V., Designing Motivation Using Persuasive Ambient Mirrors, Personal and Ubiquitous Computing, 17(1), 2013, pp. 107-126.

[34] Neeli, B.K., A Method to Engage Employees Using Gamification in BPO Industry, Third International Conference on Services in Emerging Markets, Mysore, India, Dec 12-15, 2012, pp. 142-146.

[35] Nevin, C.R., Westfall, A.O., Rodriguez, J.M., Dempsey, D.M., Cherrington, A., Roy, B., Patel, M. and Willig, J.H. Gamification as a Tool for Enhancing Graduate Medical Education, Postgraduate Medical Journal, 90(1070), 2014, pp. 685-693.

[36] Nickerson, R.C., Varshney, U. and Muntermann, J. A Method for Taxonomy Development and Its Application in Information Systems, European Journal of Information Systems, 22(3), 2013, pp. 336-359.

[37] Raftopoulos, M., Walz, S. and Greuter, S. How Enterprises Play: Towards a Taxonomy for Enterprise 
Gamification, Proceedings of DiGRA 2015: Diversity of play: Games - Cultures - Identities, 2015, pp. 1-17.

[38] Remane, G., Nickerson, R.C., Hanelt, A., Tesch, J. and Kolbe, L.M. A Taxonomy of Carsharing Business Models, 37th International Conference on Information Systems, Dublin, Ireland, Dec 11-14, 2016.

[39] Robinson, D. and Bellotti, V., A Preliminary Taxonomy of Gamification Elements for Varying Anticipated Commitment, Proceedings of the ACM CHI 2013 Workshop on Designing Gamification: Creating Gameful and Playful Experiences, 2013.

[40] Santhanam, R., Liu, D. and Shen, W. Gamification of Technology-Mediated Training: Not All Competitions Are the Same, Information Systems Research, 27(2), 2016, pp. 453-465.

[41] Sawyer, B., From Cells to Cell Processors: The Integration of Health and Video Games, IEEE Computer Graphics and Applications, 28(6), 2008,

[42] Stinson, J.N., Jibb, L.A., Nguyen, C., Nathan, P.C., Maloney, A.M., Dupuis, L.L., Gerstle, J.T., Alman, B., Hopyan, S. and Strahlendorf, C. Development and Testing of a Multidimensional Iphone Pain Assessment Application for Adolescents with Cancer, Journal of Medical Internet Research, 15(3), 2013, pp. e51.

[43] Stuart, A.G., Exercise as Therapy in Congenital Heart Disease - a Gamification Approach, Progress in Pediatric Cardiology, 38(1), 2014, pp. 37-44.

[44] Thiebes, S., Lins, S. and Basten, D. Gamifying Information Systems - a Synthesis of Gamification
Mechanics and Dynamics, European Conference on Information Systems, Tel Aviv, Istrael, Jun 9-11, 2014. [45] Thorsteinsen, K., Vitterso, J. and Svendsen, G.B. Increasing Physical Activity Efficiently: An Experimental Pilot Study of a Website and Mobile Phone Intervention, International Journal of Telemedicine and Applications, 2014, pp. 8.

[46] Van Lippevelde, W., Vangeel, J., De Cock, N., Lachat, C. Goossens, L., Beullens, K., Vervoort, L., Braet, C., Maes, L. and Eggermont, S., Using a Gamified Monitoring App to Change Adolescents' Snack Intake: The Development of the Reward App and Evaluation Design, BMC Public Health, 16(1), 2016, pp. 725 .

[47] Van Mierlo, T., Hyatt, D., Ching, A.T., Fournier, R. and Dembo, R.S. Behavioral Economics, Wearable Devices, and Cooperative Games: Results from a Population-Based Intervention to Increase Physical Activity, JMIR Serious Games, 4(1), 2016.

[48] Webster, J. and Watson, R.T., Analyzing the Past to Prepare for the Future: Writing a Literature Review, MIS Quarterly, 26(2), 2002, pp. xiii-xxiii.

[49] White, B.K., Martin, A., White, J.A., Burns, S.K., Maycock, B.R., Giglia, R.C. and Scott, J.A., TheoryBased Design and Development of a Socially Connected, Gamified Mobile App for Men About Breastfeeding (Milk Man), JMIR mHealth and uHealth, 4(2), 2016.

\section{Appendix A}

Table A-1. Taxonomy development iterations.

\begin{tabular}{|l|l|l|l|l|}
\hline Iteration & Approach & Objects & Summary & $\begin{array}{l}\text { Ending } \\
\text { Conditions }\end{array}$ \\
\hline 1 & inductive & APP-1 - APP-5 & Initial taxonomy, consisting of four dimensions & Not met \\
\hline 2 & inductive & APP-6 - APP-8 & Added three new dimensions to the initial taxonomy & Not met \\
\hline 4 & inductive & APP-9-APP-10 & Added three new dimensions & Not met \\
\hline 5 & deductive & - & $\begin{array}{l}\text { Added two new dimensions and one new } \\
\text { characteristic to an existing dimension }\end{array}$ & Not met \\
\hline 6 & inductive & APP-11-APP-13 & $\begin{array}{l}\text { Added one new dimension, merged two existing } \\
\text { dimensions, changed characteristics of two existing } \\
\text { dimensions }\end{array}$ & Not met \\
\hline 7 & inductive & APP-14-APP-17 & Added one characteristic to an existing dimension & Not met \\
\hline 8 & inductive & APP-18-APP-20 & Added one characteristic to an existing dimension & Not met \\
\hline 9 & deductive & - & Change characteristics of an existing dimension & Not met \\
\hline 10 & inductive & APP-21 - APP-23 & $\begin{array}{l}\text { No changes to taxonomy, only classification of new } \\
\text { objects }\end{array}$ & Not met \\
\hline
\end{tabular}

